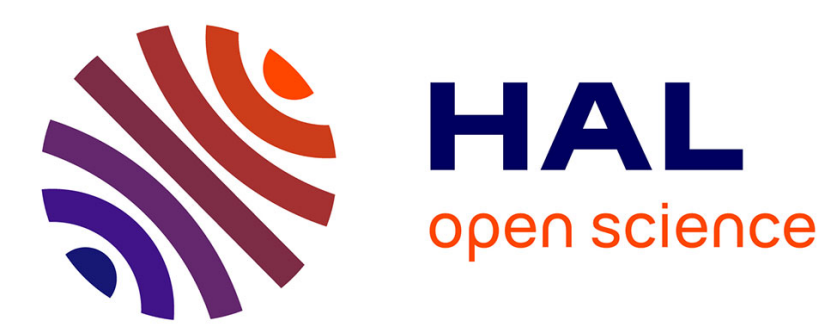

\title{
Cellules photovoltaïques utilisées en séparation spectrale - II. Couplage électrique de deux cellules solaires de type différent
}

\author{
A. Chave, J.R. Leguerre
}

\section{- To cite this version:}

A. Chave, J.R. Leguerre. Cellules photovoltaïques utilisées en séparation spectrale - II. Couplage électrique de deux cellules solaires de type différent. Revue de Physique Appliquée, 1983, 18 (9), pp.587-594. 10.1051/rphysap:01983001809058700 . jpa-00245120

HAL Id: jpa-00245120

https://hal.science/jpa-00245120

Submitted on 1 Jan 1983

HAL is a multi-disciplinary open access archive for the deposit and dissemination of scientific research documents, whether they are published or not. The documents may come from teaching and research institutions in France or abroad, or from public or private research centers.
L'archive ouverte pluridisciplinaire HAL, est destinée au dépôt et à la diffusion de documents scientifiques de niveau recherche, publiés ou non, émanant des établissements d'enseignement et de recherche français ou étrangers, des laboratoires publics ou privés. 


\title{
Cellules photovoltaïques utilisées en séparation spectrale - II. Couplage électrique de deux cellules solaires de type différent
}

\author{
A. Chave $\left({ }^{*}\right)$ et J. R. Leguerre (**) \\ Faculté des Sciences, Département de Physique, Avenue Ibn Batota, Rabat, Maroc
}

(Reçu le 17 juin 1982, révisé le 23 mars 1983, accepté le 25 mars 1983)

\begin{abstract}
Résumé. - La mise en série électrique de deux cellules solaires de type différent modifie la caractéristique couranttension de la cellule qui, isolée, possédait le plus fort courant. Si les deux cellules fonctionnent en séparation spectrale au moyen d'un miroir déchroïque, la valeur de l'énergie de coupure du miroir, au point de rendement maximum est fonction de sa pente, de la valeur des paramètres physiques des cellules et de la concentration solaire. On détermine les conditions d'optimisation du système, et en particulier la variation des paramètres de grille, du taux d'ombrage et de la température des cellules. Le module série peut fournir un rendement semblable à celui constitué de cellules isolées, mais sa sensibilité à la variation des paramètres est beaucoup plus grande.
\end{abstract}

\begin{abstract}
In the electric coupling of two different types of solar cells, the $I(V)$ characteristic, which has the more important current is modified. If the two cells are illuminated in spectral splitting with a dichroïc mirror, the cutt off energie at the point of maximum power efficiency is a function of the physical parameters values, slope of the miror and solar concentration respectively. We determine the optimization conditions of the system and in particular the variations of the grid metalization, shadowing ratio and cells temperature. The power conversion efficiency of the series coupling can be similar to the isolated cells, but shows a very high sensitivity to respect the variation of the parameters.
\end{abstract}

\section{Introduction.}

Dans la première partie de cette étude nous avons montré le comportement de deux cellules solaires couplées par un miroir déchroïque et nous avons comparé les deux couplages $\left(\mathrm{Ga}_{1-y} \mathrm{Al}_{y} \mathrm{As}\right) \mathrm{Ga}_{1-x} \mathrm{Al}_{x} \mathrm{As}$ $\mathrm{Si}$ et $\mathrm{GaAs}-\mathrm{Ge}$. Les cellules de chaque système étaient électriquement indépendantes et leurs caractéristiques électriques sans influence réciproque. Cependant dans la réalisation d'un ensemble il peut être utile de réaliser des associations en série électrique des deux cellules de manière à constituer un module unique possédant une caractéristique courant-tension propre. Dans l'étude qui suit on montrera comment se comporte l'association série de deux cellules - pour les deux couplages envisagés précédemment - par rapport à la variation des paramètres technologiques des cellules, du pourcentage molaire d'aluminium $x$ du $\mathrm{Ga}_{1-x} \mathrm{Al}_{x} \mathrm{As}$, de la concentration solaire, de la pente

(*) Adresse France : L.A.I.A.T., Université de Toulon, R.N. 98, 83130 La Garde, France.

$\left({ }^{* *}\right)$ Adresse permanente en France : L.A.A.S., 7, avenue du Colonel Roche, 31400 Toulouse, France. et de l'énergie de coupure du miroir. On redéterminera les paramètres de la grille de métallisation ainsi que la température des cellules.

\section{Modélisation de la mise en série électrique.}

Lorsque deux cellules de nature différente, utilisées dans un système à séparation spectrale sont indépendantes électriquement $\left({ }^{1}\right)$, les courants qu'elles fournissent sont fonction de l'énergie de coupure du miroir. Leur couplage électrique série, (Fig. 1), impose au module le courant de la cellule qui, montée en C.I. était le plus faible. La liaison électrique étant physiquement réalisée, il y a donc lieu de recalculer la cellule dont le courant est imposé par l'autre, donc de redéterminer sa tension de sortie, ses paramètres de grille, et sa température. En effet, pour des valeurs de $E_{\mathrm{c}}\left({ }^{2}\right)$ telles que la différence des courants des C.I.

( ${ }^{1}$ ) Afin d'éviter la répétition fréquente des expressions " couplage électrique série " et "cellules indépendantes électriquement ", on notera respectivement C.S. et C.I.

$\left(^{2}\right)$ La signification des paramètres est identique ici à celle de la première partie de l'étude. 


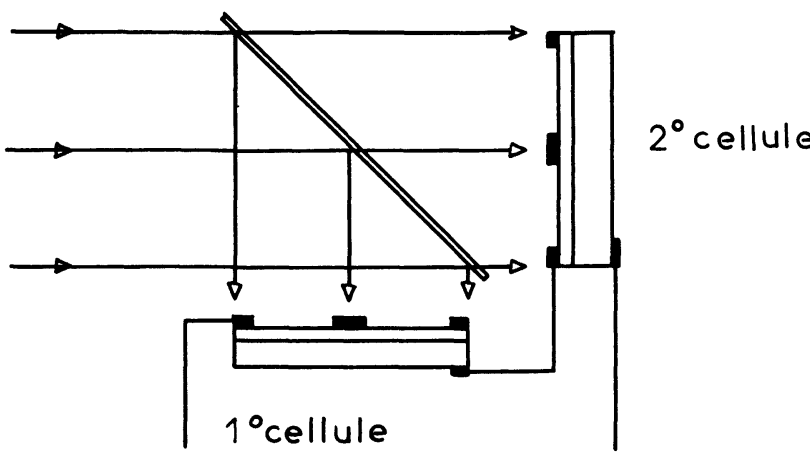

Fig. 1. - Schéma du couplage électrique série des deux cellules.

[Two cells system with serie electric coupling.]

est grande, le fait d'imposer à une cellule un courant très différent entraîne une forte augmentation de ses pertes et un échauffement de la cellule qui a pour effet de modifier ses paramètres physiques, et entraîne une dérive du photocourant, et du courant d'obscurité. Le calcul de la cellule modifiée sera donc itéré jusqu'à stabilisation de sa température. Le modèle de calcul des pertes, des paramètres de grille, et de la température est identique à celui de la première partie.

\section{Comportement du couplage série.}

On donnera simultanément les résultats obtenus pour les couplages $\left(\mathrm{Ga}_{1-y} \mathrm{Al}_{y} \mathrm{As}(\mathrm{P})\right)-\mathrm{Ga}_{1-x} \mathrm{Al}_{x} \mathrm{As}$ $\left(\mathrm{P}^{+}, \mathbf{N}\right)-\mathrm{Si}\left(\mathrm{N}^{+}, \mathrm{P}\right)$ et $\mathrm{GaAs}\left(\mathrm{P}^{+}, \mathbf{N}\right)-\mathrm{Ge}\left(\mathrm{N}^{+}, \mathrm{P}\right)$. Les valeurs des constantes physiques sont identiques à celles de la première partie.

3. INFLUENCE DE LA CONCENTRATION MOLAIRE D'ALUMINIUM $x$ SUR LE RENDEMENT DU COUPLAGE. - Les figure $2 a, 2 b$ présentent la variation du rendement des cellules en C.S. pour des valeurs de la concentration molaire d'AlAs $x$ allant de 0 à 0,70 . La valeur $E_{\mathrm{cm}}$ de $E_{\mathrm{c}}$ au rendement maximum pour $0<x<0,3$ varie peu. Pour des valeurs supérieures il y a distorsion des courbes et un fort déplacement de $E_{\mathrm{cm}}$. D'autre part, pour $x=0$ par exemple et $P=40 \mathrm{u} / \mu \mathrm{m}$ (pente du miroir), la valeur de $E_{\mathrm{cm}}$ vaut $1,81 \mathrm{eV}$ alors que pour un montage C.I. nous avions $E_{\mathrm{cm}}=1,44 \mathrm{eV}$. Remarquons tout d'abord que pour le système C.S. le rendement est maximum pour la valeur de $E_{\mathrm{c}}$ telle que si les cellules étaient montées en C.I. leurs courants au maximum de puissance $\left(I_{\max }\right)$ seraient égaux. En effet, sur le réseau de la figure 3 où l'on représente la variation de $I_{\max }$ des deux cellules indépendantes, si l'on fait décroître $E_{\mathrm{c}}$ pour une concentration unité, le courant de la cellule $\mathrm{Ga}_{1-x} \mathrm{Al}_{x} \mathrm{As}$ croît alors que celui de la cellule $\mathrm{Si}$ décroît. Au point $\mathrm{A}$ les courants sont égaux et le rendement est maximum. Toute autre valeur commune des courants sera forcément inférieure et le rendement de chaque cellule aura diminué.

Le fait que le rendement soit maximum à l'égalité des courants $I_{\max 1}$ et $I_{\max 2}$ des C.I. explique la variation de $E_{\mathrm{cm}}$ avec $x$ d'une part, d'autre part sa variation

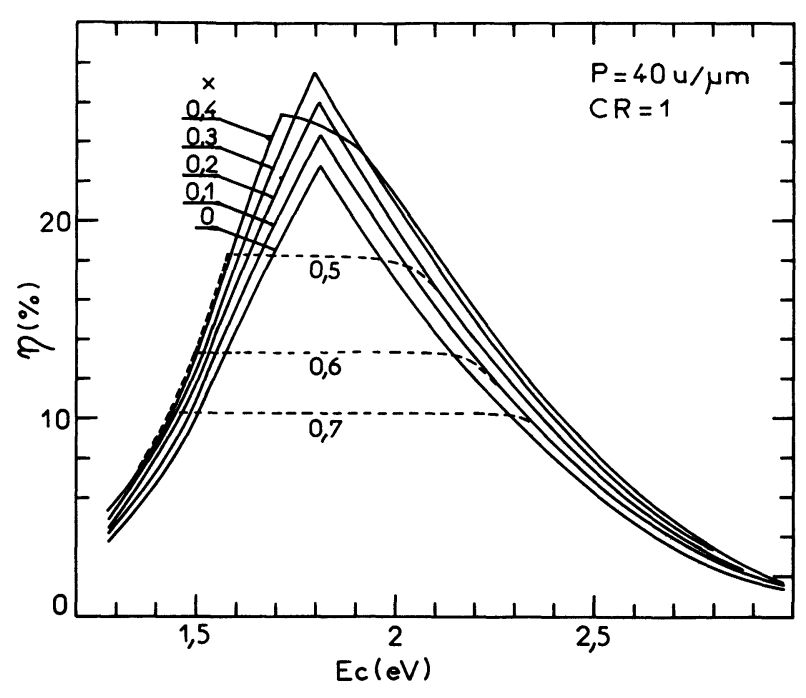

a)

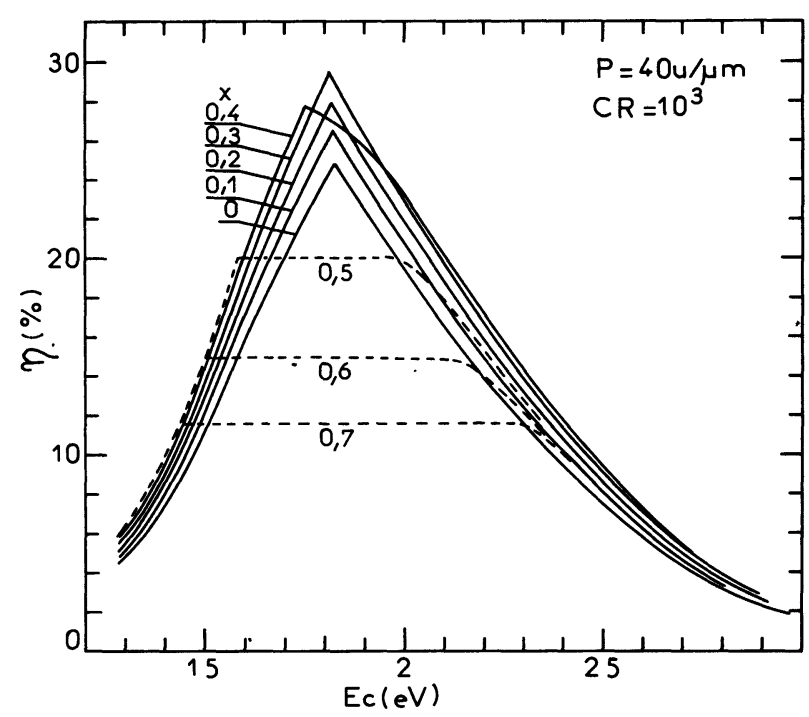

b)

Fig. $2 a, 2 b .-\mathrm{Ga}_{1-x} \mathrm{Al}_{x} \mathrm{As}\left(D H=0,1 \mu \mathrm{m} X_{1}=0,3 \mu \mathrm{m}\right.$; $\left.s_{1}=10^{4} \mathrm{~cm} / \mathrm{s}\right) . S i\left(X_{2}=0,3 \mu \mathrm{m} ; s_{2}=10^{4} \mathrm{~cm} / \mathrm{s}\right)$. Variation du rendement du système C.S. pour $0<x<0,7$ en fonction de $E_{\mathrm{c}}$.

[Power conversion efficiency for the series electric coupling when $0<x<0.7$ versus $E_{\mathrm{c}}$ ]

avec la concentration solaire. Pour $C R=1, I_{\max 2}$ du Si coupe les courbes pour $0<x<0,3$ sensiblement au même point $\mathbf{A}$ (Fig. 3) du fait du rapprochement des courbes représentant $I_{\max 1}$. Mais pour $0,4<x<0,7$ l'intersection se déplace en B, C, D, E ce qui entraîne une forte dérive de $E_{\mathrm{cm}}$.

Si l'on se place au point $C$ pour $x=0,5$ (Fig. 3), ce point se trouve sur le pallier de saturation du courant $I_{\max 1}$ et pour $E_{\mathrm{c}}<1,94 \mathrm{eV}$ le rendement $\eta_{1}$ est constant. Le courant de la cellule au Si est alors ramené à la valeur constante de celle du $\mathrm{Ga}_{1-x} \mathrm{Al}_{x} \mathrm{As}$ 
qui lui est inférieur et son rendement reste alors sensiblement constant. Alors, pour

$$
1,57 \mathrm{eV}<E_{\mathrm{c}}<1,94 \mathrm{eV}
$$

le rendement total qui est la somme des rendements partiels reste aussi constant. Pour $E_{\mathrm{c}}<1,57 \mathrm{eV}$ le rendement, entraîné par la chute de $I_{\max 2}$ décroît rapidement.

La variation de la concentration solaire entraîne un changement dans la pente des courbes $I_{\max 1,2}$ (Fig. 3) et par conséquent une modification de la valeur de $E_{\mathrm{cm}}$.

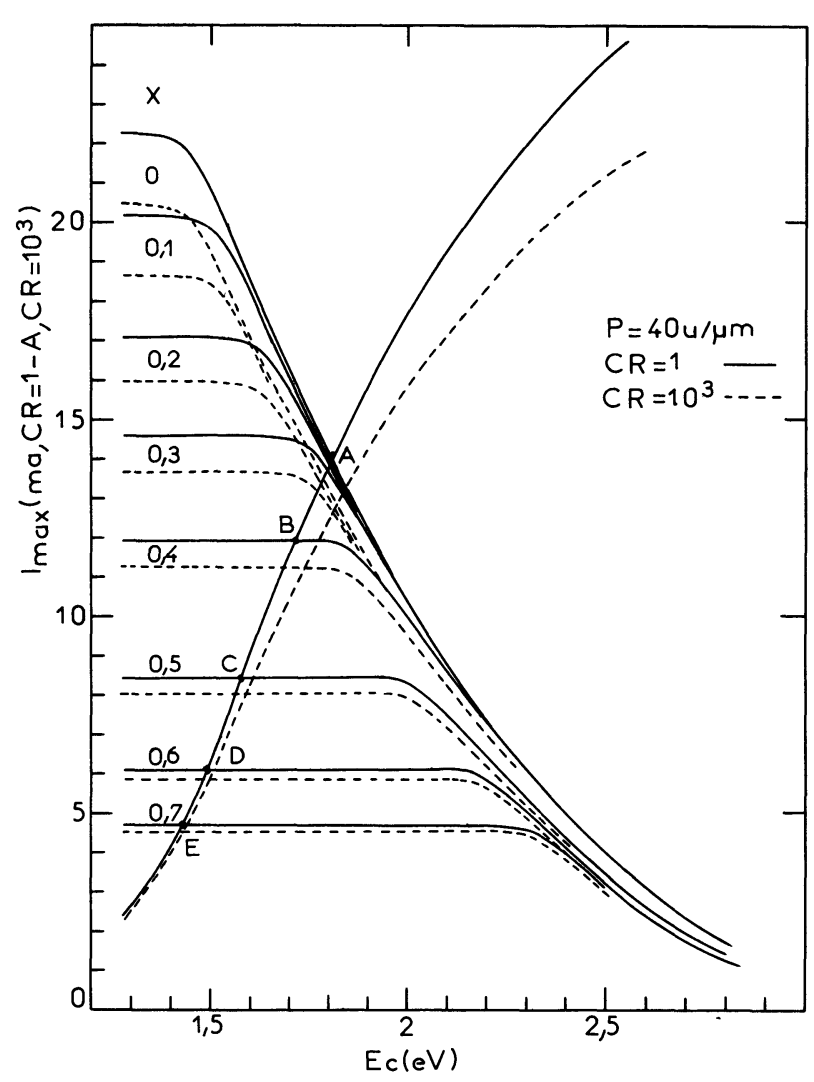

Fig. 3. - Variation des courants au maximum de puissance $I_{\max 1}$ et $I_{\max 2}$ en fonction de $E_{\mathrm{c}}$ pour $0<x<0,7$. Les paramètres des cellules sont ceux de la figure 2 .

[Variation of the currents at the power maximum versus $E_{\mathrm{c}}$ for $0<x<0.7$. The parameters of the cells are the same as those given in figure 2.]

La figure 4 donne les variations de $E_{\mathrm{cm}}$ et du rendement maximum lorsque $0<x<0,7$ pour des pentes du miroir de 5 et $40 \mathrm{u} / \mu \mathrm{m}$ et des concentrations de 1 et $10^{3}$.

\subsection{SENSIBILITÉ DE $E_{\mathrm{cm}}$ ET DU RENDEMENT À LA VARIA-} TION DES PARAMÈTRES PHYSIQUES.

3.2.1 Couplage GaAlXAs-Si. - Le paramètre $x$ est fixé à la valeur $x=0,3$ dans l'étude qui suit.

On représente sur la figure 5 la variation du ren-

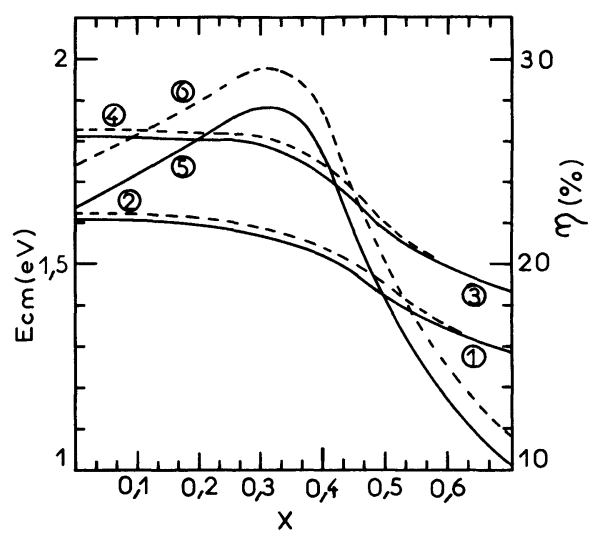

Fig. 4. $-C R=1: P=5$ ou $40 \mathrm{u} / \mu \mathrm{m}$ (courbes 1 et 3 ). $C R=10^{3}: P=5$ ou $40 \mathrm{u} / \mu \mathrm{m}$ (courbes 2 et 4 ). Courbes 5 et $6: \eta=f(x)$ lorsque $P=40 \mathrm{u} / \mu \mathrm{m}$ et pour $C R=1$ ou $10^{3}$. Variation de $E_{\mathrm{cm}}$ et $\eta$ en fonction de $x$. Les paramètres des cellules sont les mêmes que ceux de la figure 2 .

[Variation of $E_{\mathrm{cm}}$ and $\eta$ versus $x$. The cells parameters are the same as those given in the figure 2.]

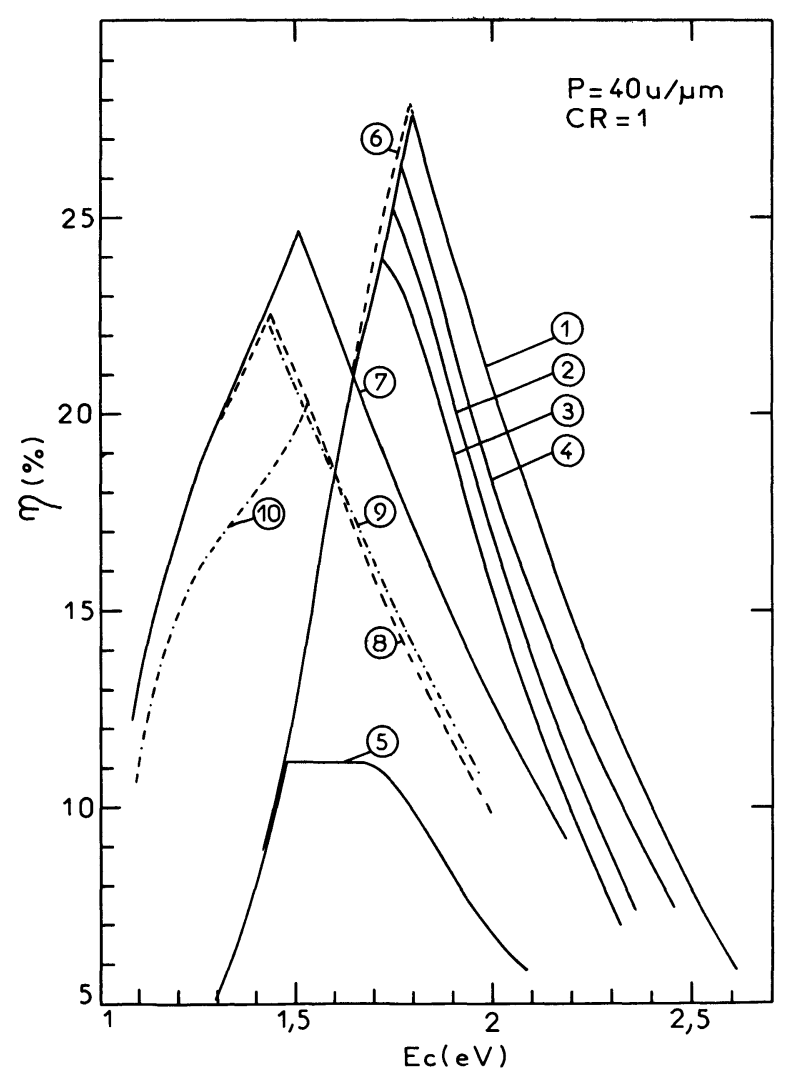

Fig. 5. - Variation de $\eta$ en fonction de $E_{\mathrm{c}}$ pour différentes valeurs de $X_{1}, X_{2}$ ou $s_{1}, s_{2} . \mathrm{Ga}_{0,7} \mathrm{Al}_{0,3} \mathrm{As}-\mathrm{Si}\left(s_{1}=s_{2}=\right.$ $\left.10^{4} \mathrm{~cm} / \mathrm{s}\right), X_{1}=X_{2}=0,3 \mu \mathrm{m}, D H=0,1 \mu \mathrm{m}(1) ; D H=$ $1 \mu \mathrm{m}(2) ; D H=2 \mu \mathrm{m}(3) ; D H=0,1 \mu \mathrm{m}, X_{1}=1 \mu \mathrm{m}$, $X_{2}=0,3 \mu \mathrm{m}(4) ; X_{1}=3 \mu \mathrm{m}, X_{2}=0,3 \mu \mathrm{m}(5) ; X_{1}=$ $0,3 \mu \mathrm{m}, X_{2}=5 \mu \mathrm{m}(6) ;$ GaAs-Ge $X_{1}=X_{2}=0,3 \mu \mathrm{m}$; $s_{1}=s_{2}=10^{4} \mathrm{~cm} / \mathrm{s}(7) ; X_{1}=3 \mu \mathrm{m}, X_{2}=0,3 \mu \mathrm{m}, s_{1}=$ $s_{2}=10^{4} \mathrm{~cm} / \mathrm{s}(8) ; X_{1}=X_{2}=0,3 \mu \mathrm{m}, s_{1}=10^{6} \mathrm{~cm} / \mathrm{s}$; $s_{2}=10^{4} \mathrm{~cm} / \mathrm{s} \quad(9) ; X_{1}=X_{2}=0,3 \mu \mathrm{m} ; s_{1}=s_{2}=$ $10^{6} \mathrm{~cm} / \mathrm{s}(10)$.

[Variation of $\eta$ versus $E_{\mathrm{c}}$ for different values of $X_{1,2}$ and $s_{1,2}$.] 
dement pour un miroir de pente de $40 \mathrm{u} / \mu \mathrm{m}$ et pour une concentration solaire unité en fonction de $E_{\mathrm{c}}$.

Si l'épaisseur $D H$ de la fenêtre augmente, $E_{\mathrm{cm}}$ se décale vers les basses énergies (courbes 1, 2, 3), ceci étant dû au fait que le courant $I_{\max 1}$ diminue lorsque $D H$ augmente, ceci entraînant un déplacement de l'intersection $I_{\max 1}\left(E_{\mathrm{c}}\right), I_{\max 2}\left(E_{\mathrm{c}}\right)$. Le rendement décroît parallèlement. Il en est de même si l'épaisseur de la zone diffusée $X_{1}$ du $\mathrm{Ga}_{1-x} \mathrm{Al}_{x} \mathrm{As}$ augmente (courbes 4, 5). Si $D H$ passe de $0,1 \mu \mathrm{m}$ à $1 \mu \mathrm{m}$ (courbes 1,2$) E_{\mathrm{cm}}$ varie de $1,81 \mathrm{eV}$ à $1,75 \mathrm{eV}$ et si $X_{1}$ passe de $0,3 \mu \mathrm{m}$ à $1 \mu \mathrm{m}$, $E_{\mathrm{cm}}$ varie de $1,81 \mathrm{eV}$ à $1,77 \mathrm{eV}$, (courbes 1,4$) . E_{\mathrm{cm}}$ est peu sensible à la variation de la zone diffusée du silicium, $X_{2}$ (courbe 6) ou à sa vitesse de recombinaison en surface, ceci provenant du fait que les courants $I_{\max 1}$ et $I_{\max 2}$ des deux cellules sont égaux dans une zone où les caractéristiques du silicium sont très peu dispersées lorsque $X_{2}$ et $s_{2}$ varient (cf. Fig. $8 a, 1^{\text {re }}$ partie).

3.2.2 Couplage GaAs-Ge. - Sur les courbes 7 et 8 de la figure 5 on remarque une variation d'amplitude du rendement et une dispersion de $E_{\mathrm{cm}}$ en fonction des paramètres $X_{1}$ et $s_{1}$ du GaAs pour $X_{1}=0,3 \mu \mathrm{m}$ constant lorsque $s_{1}$ passe de $10^{4}$ à $10^{6} \mathrm{~cm} / \mathrm{s}, E_{\mathrm{cm}}$ varie de $1,51 \mathrm{eV}$ avec $\eta=24,7 \%$ à $1,43 \mathrm{eV}$ avec $\eta=22,4 \%$ (courbes 7,9 ).

Pour $s_{1}=10^{4} \mathrm{~cm} / \mathrm{s}$ si $X_{1}$ varie de $0,3 \mu \mathrm{m}$ à $3 \mu \mathrm{m}$ (courbes 7 et 8 ), $E_{\mathrm{cm}}$ varie de $1,51 \mathrm{eV}$ avec $\eta=24,7 \%$ à $1,44 \mathrm{eV}$ avec $\eta=22,5 \%$. La sensibilité de $s_{2}$ est marquée par les courbes $(9,10)$ où $s_{2}$ passe de $10^{4}$ à $10^{6} \mathrm{~cm} / \mathrm{s}$ et $E_{\mathrm{cm}}$ varie de $1,43 \mathrm{eV}$ à $1,54 \mathrm{eV}$, le rendement allant de $22,4 \%$ à $20,2 \%$.

En résumé, $E_{\mathrm{cm}}$ est sensible aux variations de $X_{1}$ et $s_{1}$ pour le GaAs; pour le germanium si $s_{2}=10^{4} \mathrm{~cm} / \mathrm{s}$ $X_{2}$ influe peu sur $E_{\mathrm{cm}}$ par contre si $s_{2}=10^{6} \mathrm{~cm} / \mathrm{s}$, $E_{\mathrm{cm}}$ se déplace avec $s_{2}$ (cf. Fig. $8 b, 1^{\text {re }}$ partie).

3.3 INFluenCE DE LA PENTE DU MIROIR. - Pour un montage C.I., $E_{\mathrm{cm}}$ est fonction de la pente du miroir, est pratiquement indépendant de la concentration solaire (cf. Tableau I, 1 re partie). Pour le couplage C.S., comme le montre la figure $6, E_{\mathrm{cm}}$ est fonction de $P$ et de la concentration solaire. $\mathrm{Si} C R$ varie de 1 à $10^{3}$ pour $P=40 \mathrm{u} / \mu \mathrm{m}$ (courbes 2 et 5 ), $E_{\mathrm{cm}}$ passe de $1,795 \mathrm{eV}$ à $1,815 \mathrm{eV}$ pour le couplage $\mathrm{Ga}_{1-x} \mathrm{Al}_{x} \mathrm{As}-\mathrm{Si}$. Cet écart est faible, mais si l'on maintient $E_{\mathrm{cm}}$ constant lorsque $C R$ change, ceci entraînera une variation sensible du rendement que l'on examinera par la suite.

Pour le couplage GaAs-Ge pour $P=40 \mathrm{u} / \mu \mathrm{m} E_{\mathrm{cm}}$ varie de $1,43 \mathrm{eV}$ à $1,44 \mathrm{eV}$ (courbes 8 et 11).

La variation de $E_{\mathrm{cm}}$ provient d'une variation du point d'intersection des courants $I_{\max 1}$ et $I_{\max 2}$ lorsque $C R$ change, ces courants n'étant pas proportionnels à la concentration.

3.4 VARIATION DU RENDEMENT AVEC LA CONCENTRATION. - Sur la figure 7 on représente la variation du rendement en fonction de la concentration solaire

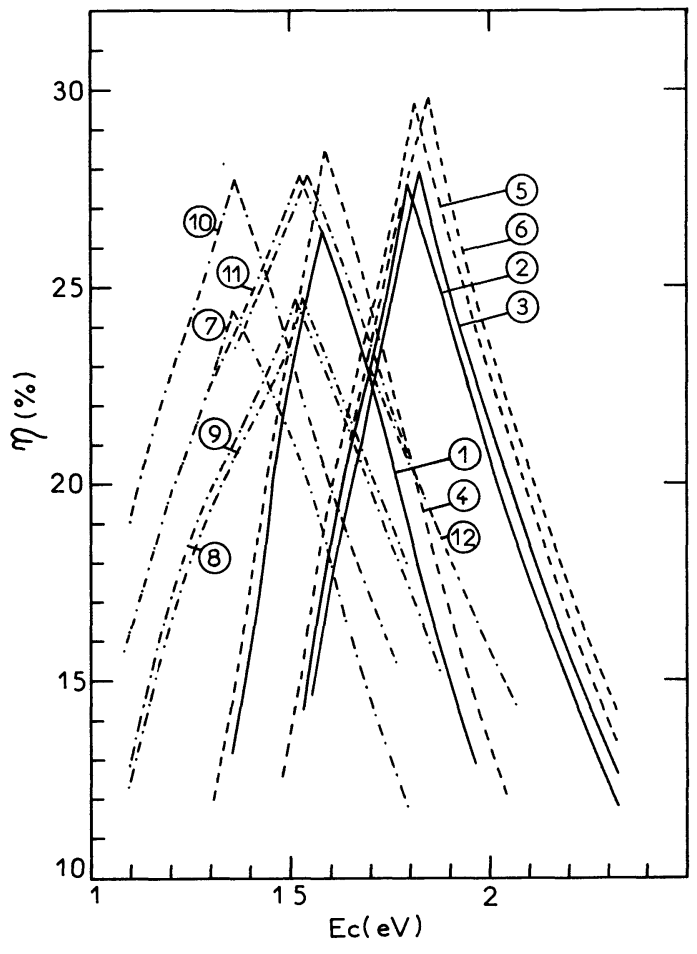

Fig. 6. - Etude du rendement en fonction de $E_{\mathrm{c}}$ lorsque la pente et la concentration varient. $\mathrm{Ga}_{0,7} \mathrm{Al}_{0,3} \mathrm{As}-\mathrm{Si}(D H=$ $\left.0,1 \mu \mathrm{m}, X_{1}=X_{2}=0,3 \mu \mathrm{m} ; s_{1}=s_{2}=10^{4} \mathrm{~cm} / \mathrm{s}\right) \mathrm{GaAs}-\mathrm{Ge}$ $\left(X_{1}=X_{2}=0,3 \mu \mathrm{m}, s_{1}=s_{2}=10^{4} \mathrm{~cm} / \mathrm{s}\right) \mathrm{Ga}_{0,7} \mathrm{Al}_{0,3} \mathrm{As}-\mathrm{Si}$ : pour des pentes de 5,40 et $10 \mathrm{C} \mathrm{u} / \mu \mathrm{m}$ : courbes $1,2,3 \mathrm{si} C R=1$ et (4), (5), (6) si $C R=10^{3}$. GaAs-Ge : Pour des pentes de 5,40 et $100 \mathrm{u} / \mu \mathrm{m}$ : courbes $7,8,9$ si $C R=1$ et (10), (11), (12) si $C R=10^{3}$.

[Power conversion efficiency versus $E_{\mathrm{c}}$ for different slopes of the mirror and different solar concentration.]

en pente $40 \mathrm{u} / \mu \mathrm{m}$ pour des couplages de cellules dont les paramètres sont les suivants :

$\mathrm{Ga}_{0,7} \mathrm{Al}_{0,3} \mathrm{As}-\mathrm{Si}$ :

$D H=0,1 \mu \mathrm{m}, X_{1}=X_{2}=0,3 \mu \mathrm{m}, s_{1}=s_{2}=10^{4} \mathrm{~cm} / \mathrm{s}$

GaAs-Ge :

$X_{1}=X_{2}=0,3 \mu \mathrm{m}, s_{1}=10^{6} \mathrm{~cm} / \mathrm{s}, s_{2}=10^{4} \mathrm{~cm} / \mathrm{s}$.

Les courbes 1 et 2 comparent les rendements du système $\mathrm{Ga}_{0,7} \mathrm{Al}_{0,3} \mathrm{As}-\mathrm{Si}$, monté respectivement en C.I. et en C.S. Pour le couplage $\mathrm{GaAs}-\mathrm{Ge}$, les courbes C.I. et C.S. sont pratiquement identiques (courbe 6). On remarque cependant que pour $C R=1$, si les cellules sont en C.I. $E_{\mathrm{cm}}=1,38 \mathrm{eV}$; et si elles sont en C.S. $E_{\mathrm{cm}}=1,43 \mathrm{eV}$ le glissement de $E_{\mathrm{cm}}$ pour obtenir l'égalité des courants $I_{\max 1}$ et $I_{\max 2}$ n'entraîne pas de chute de rendement par le fait d'une compensation entre $\eta_{1}$ et $\eta_{2}$; en effet, le glissement de $E_{\mathrm{cm}}$ vers les hautes énergies abaisse le rendement de $\eta_{1}$ et augmente $\eta_{2}$. Pour cette combinaison de cellules les deux variations se compensent.

Les courbes en C.S. sont tracées pour la valeur de $E_{\mathrm{c}}=E_{\mathrm{cm}}$ pour chaque concentration. 
$E_{\mathrm{cm}}$ se décale avec la concentration. Il y a donc lieu d'apprécier la variation du rendement en supposant $E_{\text {cm }}$ fixée à sa valeur pour $C R=1$ (courbe 5 et 9) et d'autre part de voir la chute de rendement qu'entraîne une erreur sur $E_{\mathrm{cm}}$. Nous avons choisi une erreur de $\pm 2 \%$ qui conduit aux courbes 3 et 4 pour le système $\mathrm{Ga}_{0,7} \mathrm{Al}_{0,3} \mathrm{As}-\mathrm{Si}$ et (7) et (8) pour GaAs-Ge.

Les tableaux I et II donnent les valeurs numériques pour des valeurs discrètes de la concentration.

Les courbes figure 7 ou le tableau I montrent que la perte de rendement sera moins sensible si pour travailler à une concentration quelconque on conserve la valeur de $E_{\mathrm{cm}}$ déterminée pour $C R=1$, que si l'on fait une erreur de $\pm 2 \%$ sur la valeur optimum de $E_{\mathrm{cm}}$ ceci étant valable pour les deux types de couplage mais moins sensible sur le couplage GaAs-Ge du fait de la plus faible pente des courbes $\eta\left(E_{\mathrm{c}}\right)$ au voisinage du maximum.

Afin de compléter les données sur le système série, on représente figure 8 la variation des tensions de sortie obtenues pour les cellules C.S. en fonction de la concentration solaire et on les compare aux tensions des cellules en C.I. Les valeurs de $E_{\mathrm{c}}$ sont telles que $E_{\mathrm{c}}=E_{\mathrm{cm}}$.

Dans le couplage $\mathrm{Ga}_{0,7} \mathrm{Al}_{0,3} \mathrm{As}-\mathrm{Si}$, lorsque la concentration augmente, le fait que le courant imposé à la cellule $\mathrm{Si}$ soit inférieur au courant qu'elle avait en C.I., provoque une légère augmentation de sa tension de sortie ce qui fait que $V_{\text {total }}$ (C.S.) $>V_{1}$ (C.I.) $+V_{2}$ (C.I.), (courbes 1,2 et 3 respectivement pour $\mathrm{Ga}_{0,7} \mathrm{Al}_{0,3} \mathrm{As}-\mathrm{Si}, \mathrm{Ga}_{0,7} \mathrm{Al}_{0,3} \mathrm{As}, \mathrm{Si}$ ).

Dans le couplage GaAs-Ge l'effet est peu sensible si l'on compare les deux systèmes, on peut donc voir que la stabilité du montage le plus performant est la plus faible.

\subsection{DÉTERMINATION DE LA GRILLE DE MÉTALLISATION.} Température Des Cellules. - Dans la mesure où pour le montage série les courants au maximum de puissance pour $E_{\mathrm{c}}=E_{\mathrm{cm}}$ sont différents de ceux pour le montage C.I., il y a une modification à apporter aux taux d'ombrage $S_{1,2}$ et à la distance interligne de

Tableau I. $-\mathrm{Ga}_{0,7} \mathrm{Al}_{0,3} \mathrm{As}-\mathrm{Si}-\mathrm{P}=40 \mathrm{u} / \mu \mathrm{m}$. Variation du rendement pour une erreur de $\pm 2 \%$ sur la valeur de $E_{\mathrm{cm}}$, ou $E_{\mathrm{cm}}$ fixée à sa valeur pour $C R=1$.

[Conversion efficiency for a variation of $\pm 2 \%$ of the $E_{\mathrm{cm}}$ value, or $E_{\mathrm{cm}}$ fixed at the value for $C R=1$.]

\begin{tabular}{|c|c|c|c|c|c|c|c|}
\hline \multirow{2}{*}{$C R$} & $E_{\mathrm{cm}}(\mathrm{eV})$ & $E_{\mathrm{cm}}+2 \%$ & $E_{\mathrm{cm}}-2 \%$ & $E_{\mathrm{c}}(C R=1)$ & \multirow{2}{*}{$\begin{array}{c}\Delta \eta \\
(+2 \%)\end{array}$} & \multirow{2}{*}{$\begin{array}{c}\Delta \eta \\
(-2 \%)\end{array}$} & \multirow{2}{*}{$\Delta \eta\left(E_{\mathrm{c}}=E_{\mathrm{cm}}(C R=1)\right)$} \\
\hline & $\eta \%$ & $\eta \%$ & $\eta \%$ & $\eta \%$ & & & \\
\hline \multirow{2}{*}{1} & 1,795 & 1,831 & 1,759 & 1,795 & \multirow{2}{*}{$1 \%$} & \multirow{2}{*}{$0,8 \%$} & \\
\hline & 27,6 & 26,6 & 26,8 & 27,6 & & & \\
\hline \multirow{2}{*}{25} & 1,80 & 1,836 & 1,764 & 1,795 & \multirow{2}{*}{1,3} & \multirow{2}{*}{2,2} & \multirow{2}{*}{0,5} \\
\hline & 28,7 & 27,4 & 26,5 & 28,2 & & & \\
\hline \multirow{2}{*}{50} & 1,81 & 1,846 & 1,773 & 1,795 & \multirow{2}{*}{1,45} & \multirow{2}{*}{2,15} & \multirow{2}{*}{0,75} \\
\hline & 29,05 & 27,6 & 26,9 & 28,3 & & & \\
\hline \multirow{2}{*}{100} & 1,81 & 1,846 & 1,764 & 1,795 & \multirow{2}{*}{1,40} & \multirow{2}{*}{2,2} & \multirow{2}{*}{0,90} \\
\hline & 29,4 & 28 & 27,2 & 28,5 & & & \\
\hline \multirow{2}{*}{400} & 1,815 & 1,851 & 1,778 & 1,795 & \multirow{2}{*}{1,43} & \multirow{2}{*}{2,08} & \multirow{2}{*}{1,08} \\
\hline & 29,68 & 28,25 & 27,6 & 28,6 & & & \\
\hline \multirow{2}{*}{$10^{3}$} & 1,815 & 1,851 & 1,778 & 1,795 & \multirow{2}{*}{140} & \multirow{2}{*}{1,90} & \multirow{2}{*}{1,10} \\
\hline & 29,6 & 28,2 & 27,7 & 28,5 & & & \\
\hline \multirow{2}{*}{$2 \times 10^{3}$} & 1,82 & 1,856 & 1,783 & 1,795 & \multirow{2}{*}{1,40} & \multirow{2}{*}{1,70} & \multirow{2}{*}{1,20} \\
\hline & 29,2 & 27,8 & 27,5 & 28 & & & \\
\hline
\end{tabular}


Tableau II. - GaAs-Ge-P $\rightleftharpoons 40 \mathrm{u} / \mu \mathrm{m}$. Variation du rendement pour une erreur de $\pm 2 \%$ sur la valeur de $E_{\mathrm{cm}}$ ou $E_{\mathrm{cm}}$ fixée à sa valeur pour $C R=1$.

[Conversion efficiency for a variation of $\pm 2 \%$ of the $E_{\mathrm{cm}}$ value, or $E_{\mathrm{cm}}$ fixed at the value for $C R=1$.]

\begin{tabular}{|c|c|c|c|c|c|c|c|}
\hline \multirow{2}{*}{$C R$} & $E_{\mathrm{cm}}(\mathrm{eV})$ & $E_{\mathrm{cm}}+2 \%$ & $E_{\mathrm{cm}}-2 \%$ & $E_{\mathrm{c}}(C R=1)$ & \multirow{2}{*}{$\begin{array}{c}\Delta \eta \\
(+2 \%)\end{array}$} & \multirow{2}{*}{$\begin{array}{c}\Delta \eta \\
(-2 \%)\end{array}$} & \multirow{2}{*}{$\Delta \eta\left(E_{\mathrm{c}}=E_{\mathrm{cm}}(C R=1)\right)$} \\
\hline & $\eta \%$ & $\eta \%$ & $\eta \%$ & $\eta \%$ & & & \\
\hline \multirow{2}{*}{1} & 1,43 & 1,458 & 1,401 & 1,43 & \multirow{2}{*}{0,7} & \multirow{2}{*}{0,6} & \\
\hline & 22,4 & 21,7 & 21,8 & 22,4 & & & \\
\hline \multirow{2}{*}{25} & 1,43 & 1,458 & 1,401 & 1,43 & \multirow{2}{*}{0,6} & \multirow{2}{*}{0,9} & \multirow{2}{*}{0,0} \\
\hline & 24,2 & 23,6 & 23,4 & 24,2 & & & \\
\hline \multirow{2}{*}{50} & 1,43 & 1,458 & 1,401 & 1,43 & \multirow{2}{*}{0,5} & \multirow{2}{*}{0,6} & \multirow{2}{*}{0,0} \\
\hline & 24,6 & 24,1 & 23,9 & 24,6 & & & \\
\hline \multirow{2}{*}{100} & 1,435 & 1,463 & 1,406 & 1,43 & \multirow{2}{*}{0,58} & \multirow{2}{*}{0,28} & \multirow{2}{*}{0,18} \\
\hline & 25,08 & 24,5 & 24,3 & 24,9 & & & \\
\hline \multirow{2}{*}{100} & 1,44 & 1,468 & 1,411 & 1,43 & \multirow{2}{*}{0,6} & \multirow{2}{*}{0,7} & \multirow{2}{*}{0,3} \\
\hline & 25,5 & 24,9 & 24,8 & 25,2 & & & \\
\hline \multirow{2}{*}{$10^{3}$} & 1,44 & 1,468 & 1,411 & 1,43 & \multirow{2}{*}{0,5} & \multirow{2}{*}{0,8} & \multirow{2}{*}{0,2} \\
\hline & 25,4 & 24,9 & 24,7 & 25,2 & & & \\
\hline \multirow{2}{*}{$2 \times 10^{3}$} & 1,44 & 1,468 & 1,411 & 1,43 & \multirow{2}{*}{0,45} & \multirow{2}{*}{0,65} & \multirow{2}{*}{0,15} \\
\hline & 25,15 & 24,7 & 24,5 & 25,0 & & & \\
\hline
\end{tabular}

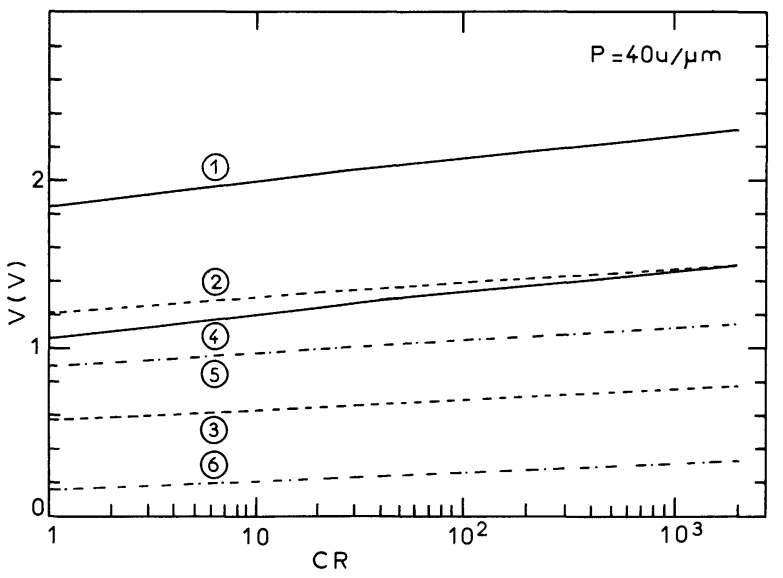

Fig. 7. - Rendement des deux systèmes en fonction de la concentration solaire. Pour la cellule GaAs : $s_{1}=10^{6} \mathrm{~cm} / \mathrm{s}$. Les autres paramètres sont ceux de la figure 6. $\mathrm{Ga}_{0,7} \mathrm{Al}_{0,3} \mathrm{As}$ : (1) système C.I. ; (2) système C.S. Si $E_{\mathrm{cm}}$ augmente de $2 \%$ : courbe 3 , s'il diminue de $2 \%$ : courbe 4 . Si $E_{\mathrm{c}}=E_{\mathrm{cm}}(C R=1)$ : courbe 5 . GaAs-Ge : (6) système C.I.; (6) système C.S. Si $E_{\mathrm{cm}}$ augmente de $2 \%$ : courbe 7 , s'il diminue de $2 \%:$ courbe 8 . Si $E_{\mathrm{c}}=E_{\mathrm{cm}}(\overline{C R=1)}$ : courbe 9 .

[Power conversion efficiency of the two systems versus the concentration. For the GaAs cell $: s_{1}=10^{6} \mathrm{~cm} / \mathrm{s}$. The others parameter of the cells are the same as those given in figure 6.] 
grille $2 L_{\mathbf{D} 1,2}$ de telle sorte que les pertes restent minimales (cf. Calcul des pertes $1^{\text {re }}$ partie).

Les variations de $S$ et $L_{\mathrm{D}}$ sont représentées sur la figure 9 en fonction de la concentration solaire. Pour chaque point la valeur de $E_{\mathrm{c}}$ est choisie optimale et égale à $E_{\mathrm{cm}}$. On compare les valeurs obtenues pour les montages C.S. et C.I.

Pour les cellules ayant le " gap » le plus élevé, soit $\mathrm{Ga}_{0,7} \mathrm{Al}_{0,3} \mathrm{As}$ et $\mathrm{GaAs}$, le taux d'ombrage $S$ diminue dans le couplage C.S. par rapport au couplage C.I. (courbes 1, 2, 6, 8).

Pour le silicium, il n'y a pratiquement pas de changement, celui du germanium augmente.

Les variations de $L_{\mathrm{D}}$ sont évidemment inversées par rapport à celles de $S$ (courbes $3,4,10,11,12,13$ ).

Les figures $10 a$ et $10 b$ montrent la variation des températures comparées des deux systèmes C.S. et C.I. pour différents radiateurs. Les températures des cellules sont calculées suivant le modèle donné dans la première partie.

\section{Conclusion.}

L'étude analytique du couplage électrique de deux cellules de type différent, fonctionnant en séparation spectrale a permis de mettre en évidence le comportement du module équivalent aux deux cellules et notamment a montré :

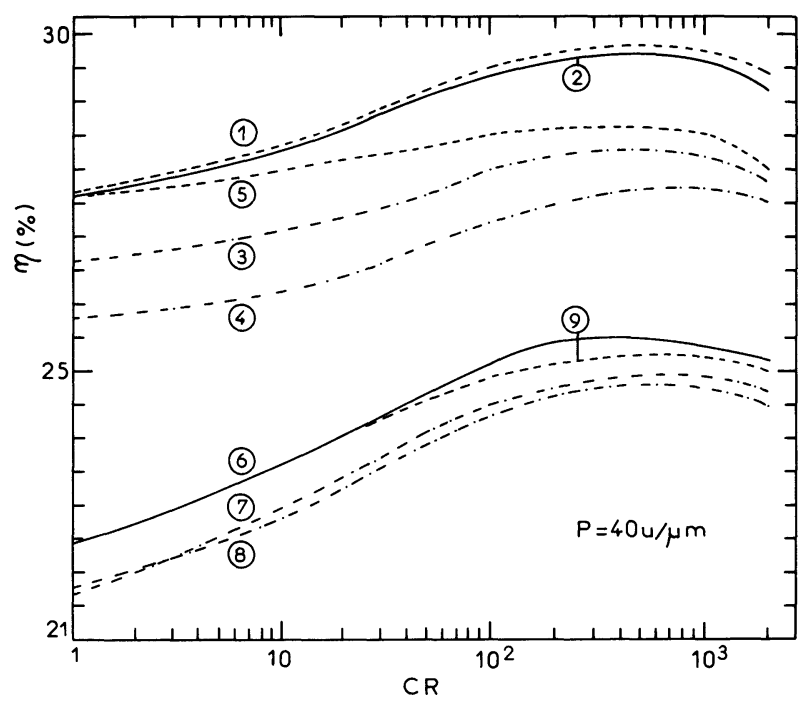

Fig. 8. - Variation des tensions de sortie pour les deux systèmes série en fonction de la concentration. On les compare aux valeurs des tensions des cellules indépendantes.

(1) Tension de sortie du couplage série $\mathrm{Ga}_{0,7} \mathrm{Al}_{0,3} \mathrm{As}-\mathrm{Si}$.

(2) Tension de sortie du $\mathrm{Ga}_{0,7} \mathrm{Al}_{0,3}$ As. (3) Si. (4) Tension de sortie du couplage série GaAs-Ge. (5) GaAs. (6) Ge. Les paramètres des cellules sont ceux de la figure 7 .

[Output voltage of the two serie electric-coupling systems versus the solar concentration. The voltages of the independant cells are plotted on the same figure. The parameters cells are the same as those given in figure 7.$]$
- que le système $\mathrm{Ga}_{1-x} \mathrm{Al}_{x} \mathrm{As}-\mathrm{Si}$ pourvu d'une " fenêtre » $\mathrm{Ga}_{0,1} \mathrm{Al}_{0,9} \mathrm{As}(\mathrm{P})$ est toujours optimale pour $x=0,3$,

- que la valeur de l'énergie de coupure du miroir $E_{\mathrm{cm}}$ au point de rendement maximum est fonction des paramètres physiques de la cellule, de la concentration solaire et de la pente du miroir,

- que les valeurs du taux d'ombrage, des paramètres de grille et de la température sont modifiés par rapport aux valeurs du couplage C.I.,

- qu'une variation de $\pm 2 \%$, de $E_{\mathrm{cm}}$ peut entraîner une chute du rendement de $2 \%$ du couplage $\mathrm{Ga}_{0,7} \mathrm{Al}_{0,3} \mathrm{As}-\mathrm{Si}$ et environ $0,6 \%$ pour GaAs-Ge.

Moyennant la connaissance de ces données, il apparaît possible de réaliser un couplage série des cellules qui aurait un rendement sensiblement identique à celui obtenu pour des cellules indépendantes mais dont la stabilité serait d'autant plus faible que son optimisation serait réalisée.

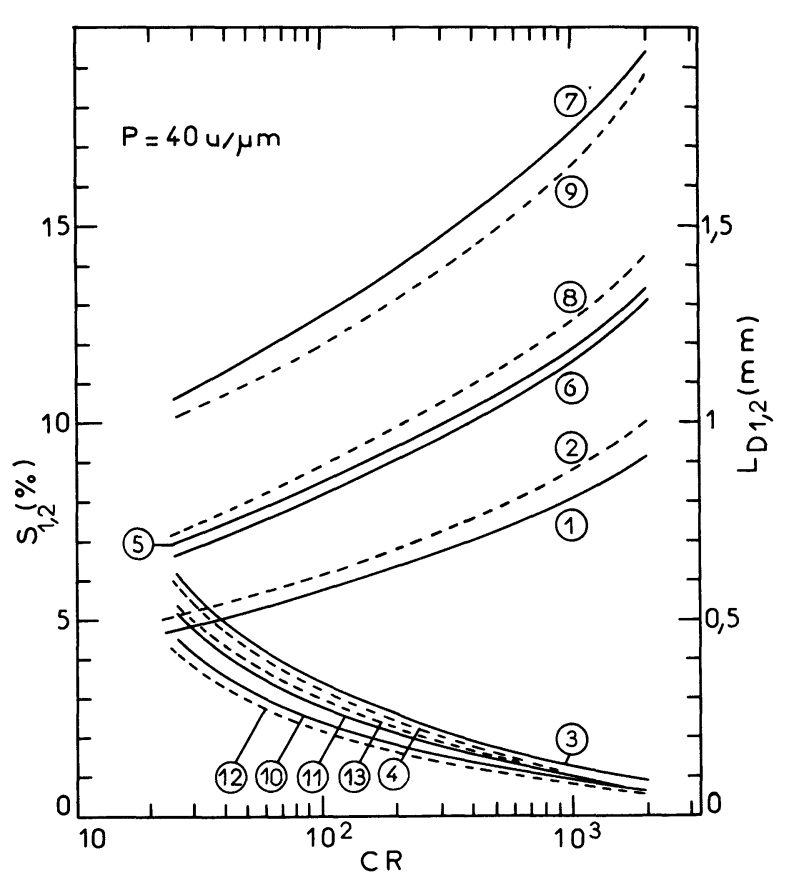

Fig. 9. - Comparaison des taux d'ombrage $S_{1,2}$ et des paramètres de grille $L_{\mathrm{D} 1,2}$ pour les systèmes C.I. et C.S. Les paramètres des cellules sont ceux de la figure 6. Pour le montage C.S., les courbes 1, 5, 6, 7 représentent $S$ et (3), (10), (10), (11) représentent $L_{\mathrm{D}}$ respectivement pour les cellules $\mathrm{Ga}_{0,7} \mathrm{Al}_{0,3} \mathrm{As}, \mathrm{Si}$, GaAs et Ge. Pour le montage C.I., $S$ est représenté par (2), (5), (8), (9) et $\mathrm{L}_{\mathrm{D}}$ par (4), (12), (12), (13) respectivement pour les cellules $\mathrm{Ga}_{0.7} \mathrm{Al}_{0.3} \mathrm{As}, \mathrm{Si}, \mathrm{GaAs}$ et $\mathrm{Ge}$.

[We compare the shadowing ratio $S_{1,2}$ and the grid parameter $L_{\mathbf{D} 1,2}$ for the two systems C.I. and C.S. when the solar concentration varies from 1 to $10^{3}$. The parameters of the cells are identical to those given in figure 6.] 


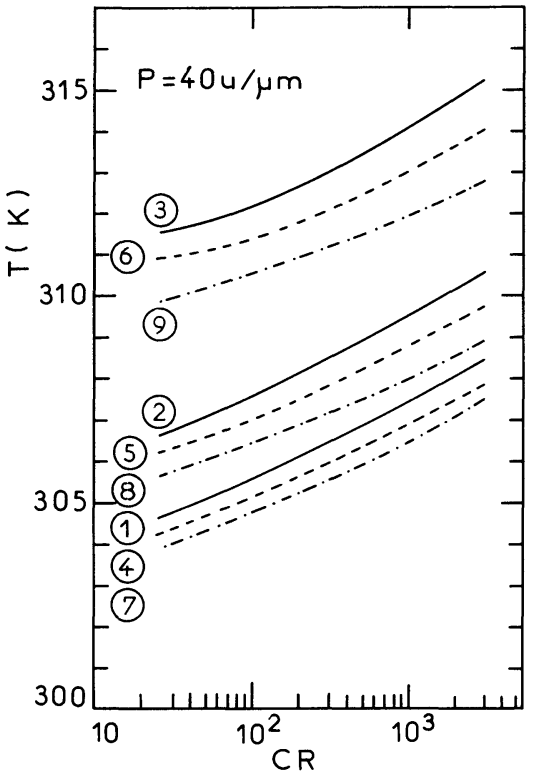

a)

Fig. $10 a$ et $10 b$. Etude comparée des températures des cellules pour les systèmes C.S. et C.I. en fonction de la concentration solaire pour différents rayons du radiateur. Les paramètres des cellules sont ceux de la figure 6. Sur la figure $10 a: \mathrm{Ga}_{0,7} \mathrm{Al}_{0,3} \mathrm{As}$ : pour $R F=25,15,10 \mathrm{~cm}$ courbes 1 , 2, 3, couplage C.S. et (4), (5), (6) montage C.I. Si : pour $R F=25,15,10 \mathrm{~cm}$, courbes $7,8,9$, inchangées en C.I. ou C.S. Sur la figure $10 b: \mathrm{GaAs}$ : pour $R F=25,15,10 \mathrm{~cm}$ :

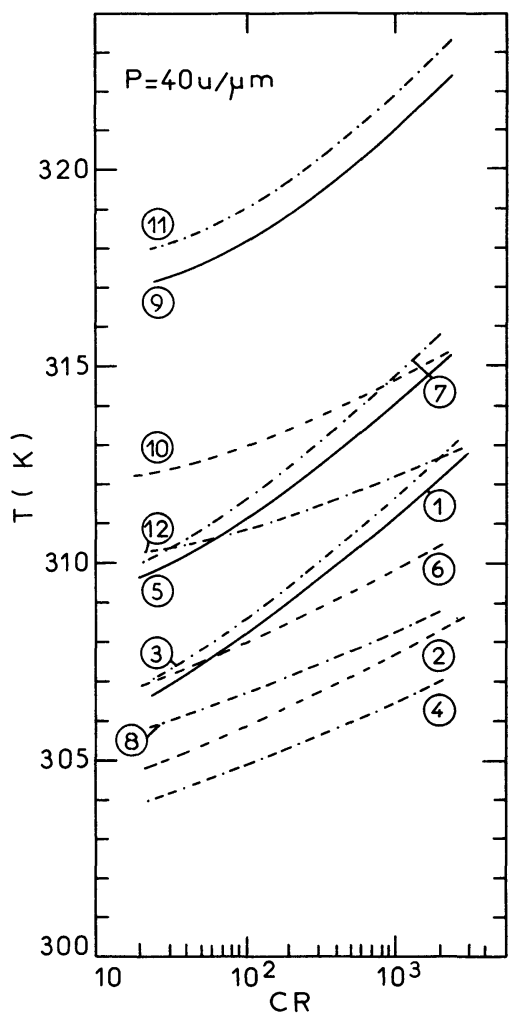

b)

courbes 1, 5, 9 couplage C.S. et (3), (7), (11) montage C.I. Ge : pour $R F=25,15,10 \mathrm{~cm}$ : courbes 2, 6, 10 couplage C.S. et (4), (8), (12) montage C.I.

[Comparative study of the cells temperature for the two systems C.S. and C.I. versus the solar concentration for different radius of the heat exchanger. The parameters of the cells are the same as those given in figure 6.] 